models. Tumors assigned to the basal-like, $\mathrm{HER}^{+}$and $\mathrm{ER}^{-}$, and luminal B intrinsic subtypes were nearly all anticipated to have a poor outcome by the 70-gene, recurrence-score and wound-response models. In addition, the outcome predictions of all five models correlated with tumor grade. Notably, the two models just beginning to be taken into a clinical setting - the 70-gene profile and recurrence score-showed $77-81 \%$ agreement in outcome prediction.

The researchers conclude that four of the five models examined produce largely concordant predictions for breast cancer because they all trace similar changes in the underlying cellular phenotype, despite using different sets of genes to do so.

Original article Fan C et al. (2006) Concordance among gene-expression-based predictors for breast cancer. $\mathrm{NEnglJ}$ Med 355: 560-569

\section{Calculating the risk of toxicity for patients with advanced GIST treated with imatinib}

Treatment with imatinib has improved 2-year survival in patients with advanced gastrointestinal stromal tumors (GIST) from less than $20 \%$ to around $70 \%$. Despite reports that imatinib is well tolerated in clinical trials, doses are often reduced because of dose-dependent toxicities such as nausea, anemia, edema, fatigue, and rash.

In an EORTC-ISG-AGITG study, 946 patients with GIST were randomized to receive imatinib at either standard (400 mg once daily) or high (400 mg twice daily) dose, with a cross-over to high dose from the standard-dose group in cases of disease progression. Factors prognostic of toxicity were investigated.

In multivariate analyses, anemia was found to correlate with imatinib dose and baseline hemoglobin level ( $P<0.0001$ for both), and neutropenia with baseline neutrophil count and hemoglobin level ( $P<0.0001$ and $P=0.0022$, respectively). For nonhematologic toxicities, risk was dosedependent. Individual toxicities were associated with patient-specific factors including sex (higher risk of edema, nasea and diarrhea in females), advanced age (edema, rash and fatigue), prior chemotherapy (fatigue) and small lesions (rash).

Based on these analyses, the authors propose a multivariate risk calculator, available on the internet (http://www.eortc.be/tools/ imatinibtoxicity), that can be used in the clinic to identify the risk of the main toxic events for individual patients with GIST treated with imatinib.

Original article Van Glabbeke M et al. (2006) Predicting toxicities for patients with advanced gastrointestinal stromal tumours treated with imatinib: a study of the European Organisation for Research and Treatment of Cancer, the Italian Sarcoma Group, and the Australasian Gastro-Intestinal Trials Group (EORTC-ISG-AGITG). Eur J Cancer 42: 2277-2285

\section{New European guidelines for the use of G-CSF for chemotherapy- induced neutropenia}

Chemotherapy-induced neutropenia is a major risk factor for infection-related morbidity and mortality and an important dose-limiting toxicity in cancer treatment. The incidence of severe neutropenia or febrile neutropenia (FN) can be reduced by prophylactic treatment with granulocytecolony stimulating factors (G-CSFs), but how such medication is used varies widely in clinical practice. The European Organisation for Research and Treatment of Cancer (EORTC) has recently produced guidelines for the use of G-CSF in adults with lymphomas and solid tumors undergoing chemotherapy.

Recommendations include the evaluation of patient-related adverse risk factors (e.g. age $\geq 65$ years, advanced disease, previous history of FN) before each cycle of chemotherapy. Prophylactic G-CSF is recommended in chemotherapy regimens associated with $\mathrm{FN}$ in more than $20 \%$ of patients. For regimens associated with $\mathrm{FN}$ in $10-20 \%$ of patients, patient-related risk factors that could increase the overall risk of FN should be carefully assessed, and those patients with an overall individual risk of $>20 \%$ for FN should also receive G-CSF. Prophylactic G-CSF support is also recommended in situations in which dose-dense or dose-intense chemotherapy strategies have survival benefits, and to support chemotherapy for which reductions in dose intensity or density are associated with a poor prognosis. Filgrastim, lenograstim and pegfilgrastim have all shown clinical efficacy and are all recommended for the prevention of FN or FN-related complications.

\footnotetext{
Original article Aapro MS et al. (2006) EORTC guidelines for the use of granulocyte-colony stimulating factor to reduce the incidence of chemotherapy-induced febrile neutropenia in adult patients with lymphomas and solid tumours. Eur J Cancer 42: 2433-2453
} 